\title{
Impact of Blood Glucose Levels on Contrast Induced Nephropathy after Percutaneous Coronary Intervention in Patients not known to be Diabetic with Acute Coronary Syndrome.
}

\author{
N Islam, ${ }^{1}$ AAS Majumder, ${ }^{1}$ M Khalequzzaman, ${ }^{1}$ M Akhtaruzzaman, ${ }^{1}$ AK Choudhury, ${ }^{1}$ MS Ali, ${ }^{2}$ \\ MR Shikder, ${ }^{1}$ S Hasem, ${ }^{1}$ ABMN Nobi, ${ }^{1}$ MS Kabir ${ }^{1}$ \\ ${ }^{1}$ Dept. of Cardiology, NICVD, Dhaka, ${ }^{2}$ IDH, Mohakhali, Dhaka.
}

\begin{abstract}
Key words:
Blood glucose,

Contrast

induced

nephropathy, Acute coronary syndrome, PCI.
\end{abstract}

\begin{abstract}
:
Background: Contrast-Induced Nephropathy (CIN) is an iatrogenic disorder, resulting from exposure to contrast media. The association between pre-procedural blood glucose levels and CI-AKI risk (regardless of pre-existing diabetes) is unknown. The present study was conducted to evaluate the incidence of CI-AKI in patients with admission hyperglycemia in non-diabetic ACS patients.

Methods: This is Prospective, observational study done in the department of Cardiology, National Institute of Cardiovascular Diseases (NICVD), Dhaka. Considering inclusion and exclusion criteria, 50 patients were non diabetic with ACS with normal blood glucose (d" $7.8 \mathrm{mmol} / \mathrm{lor}$ d" $140 \mathrm{mg} / \mathrm{dl}$ ) in (Group I) and 50 patients were non diabetic with ACS with high blood glucose ( $>7.8 \mathrm{mmol} / \mathrm{l}$ or $>140 \mathrm{mg} / \mathrm{dl}$ ) undergoing percutaneous coronary intervention in (Group II). On admission random blood glucose was measured. Non- ionic low osmolar contrast agents (lopamidol) was used in all patients. Serum creatinine, serum electrolytes was measured and creatinine clearance rate was determined within 24 hours before PCI and day 1 and 2 after PCI.

Results: The incidence of CIN was $24 \%$ in high blood glucose group and $4 \%$ in normal blood glucose group $(p=0.004)$. It was also observed that gradual incremental increase in risk of CIN associated with higher admission blood glucose level. There was positive correlation between s. creatinine and admission blood glucose but it showed negative correlation between CCr and admission blood glucose after PCI in ACS patients not known to be diabetic.

Conclusion: The present study reveals that index admission high blood glucose in acute coronary syndrome patients not known to be diabetic is associated with increased incidence of contrast induced nephropathy after percutaneous coronary intervention.
\end{abstract}

(Cardiovasc. j. 2013; 6(1): 23-30)

\section{Introduction:}

Contrast induced nephropathy is a complex syndrome of acute renal failure occurring after the administration of iodinated contrast media. ${ }^{1}$ Contrast induced nephropathy is generally defined as an increase in serum creatinine concentration of $>0.5 \mathrm{mg} / \mathrm{dl}$ ( $>44 \mu \mathrm{mol} /$ L) or $25 \%$ above baseline within 48 hours after contrast administration. ${ }^{2}$ Most recently, the acute kidney injury network has defined contrast- induced acute kidney injury(CI-AKI) as a rise in the serum creatinine level $\geq 0.3 \mathrm{mg} /$ $\mathrm{dl}$ or an increase in the serum creatinine level of $\geq 50 \%$ or more from baseline that occurred within 48 hour after coronary angiography. ${ }^{3}$ The use of iodinated contrast media has been described as the third most common cause of hospital acquired renal insufficiency. It commonly occurring after coronary angiography and/or angioplasty and computed tomography scans. ${ }^{4}$ It occurs within 24-48 hours of exposure, with creatinine level typically peaking 3-5 days after procedure and returning to baseline or near baseline value in 1-3 weeks. $^{1}$

Patients either with or without a prior history of diabetes mellitus (DM) may present with hyperglycemia during acute coronary syndrome. Among patients with no prior history of DM, hyperglycemia may reflect previously undiagnosed diabetes, pre-existing carbohydrate

Address of correspondence: Dr. Nighat Islam, Department of Cardiology, National Institute of Cardiovascular Diseases, Dhaka, Bangladesh. 
intolerance, stress-related carbohydrate intolerance, or a combination of these. ${ }^{5} \mathrm{~A}$ higher percentage of the hyperglycemic non-diabetic suffered cardiac arrest before admission compared with hyperglycemic DM (15\% and $2 \%$ respectively). ${ }^{6} \mathrm{~A}$ recent report showed that, these patients who had no known diabetes at the time of acute myocardial infarction (AMI) and whose admission blood glucose levels were less than $200 \mathrm{mg} / \mathrm{dL}(<11.1 \mathrm{mmol} / \mathrm{L})$, up to $40 \%$ were diagnosed as having impaired glucose tolerance and $25 \%$ as having diabetes when tested 3 months after discharge. ${ }^{7}$

The incidence of radiographic contrast agentinduced acute renal failure is estimated to be as high as $5.7 \%$ to $29.4 \%$ among patients with diabetes mellitus and $14.8 \%$ to $55 \%$ in patients with chronic renal insufficiency, though the incidence is $<2 \%$ in general population. ${ }^{8}$ Shaheen has shown that, the overall incidence of contrast induced nephropathy (CIN) is $10 \%$. In subgroup analysis the incidence was $13.3 \%, 10 \%$ and $20 \%$ respectively in patients with pre existing renal impairment, diabetes and both. This study also found two other risk factors for namely age and contrast volume. ${ }^{9}$ There is a decrease in the incidence of CIN when low osmolar contrast media (LOCM) are used instead of high osmolar contrast media (HOCM). ${ }^{10}$ The incidence of nephrotoxicity is less in iso-osmolar non-ionic contrast medium (e.g Iodixanol) than in lowosmolar non-ionic contrast medium (e.g Iopamidol). ${ }^{11}$ But one study by Hossain in Bangladesh has shown that no significant difference between iso-osmolar and low-omolar contrast media in patients with chronic kidney disease(CKD). ${ }^{12}$ The lowest rate of CIN occurring in patients receiving less than 100 to $140 \mathrm{ml}$ of contrast media. Contrast volume in excess of 5 $\mathrm{ml} / \mathrm{kg}$ strongly predict nephropathy requiring dialysis. A significantly increased risk of CIN has also been demonstrated among patients who received a second dose of contrast media within 48 hours. ${ }^{13}$ The most important risk marker for nephropathy after exposure to iodinated contrast media is pre-existing renal impairment and diabetes mellitus. Other markers associated with an increased risk of contrast induced nephropathy (CIN) include nephrotoxic drugs, anemia, age older than 70 years, pre-procedural hemodynamic instability, volume depletion, congestive heart failure (CHF) and hypoalbuminaemia. ${ }^{14}$ Another study by Akhtaruzzaman has shown that, the incidence of contrast induced nephropathy in anemic patients is more (26\%) after percutaneous coronary intervention (PCI), than with normal hemoglobin $(8 \%) .{ }^{15}$ However, while diabetes is a well recognized risk factor for CI-AKI, the association between pre-procedural blood glucose levels and CI-AKI risk (regardless of pre-existing diabetes) is unknown. Thus, it is possible that a combination of admission hyperglycemia in nondiabetic ACS patients and contrast exposure during PCI could significantly increase the risk for CI-AKI. ${ }^{17}$

\section{Methodology:}

This is prospective, observational study done in the department of Cardiology, National Institute of Cardiovascular Diseases (NICVD), Dhaka from July, 2011 to May, 2012. The main objective of the study was to determine the relationship between level of admission blood glucose and contrast induced nephropathy after percutaneous coronary intervention in acute coronary syndrome patients not known to be diabetic. Considering inclusion and exclusion criteria, 50 patients were non diabetic with ACS with normal blood glucose $(\leq 7.8 \mathrm{mmol} / \mathrm{l}$ or $\leq 140$ $\mathrm{mg} / \mathrm{dl}$ ) undergoing percutaneous coronary intervention (Group I) and 50 patients were non diabetic with ACS with high blood glucose ( $>7.8$ $\mathrm{mmol} / \mathrm{l}$ or $>140 \mathrm{mg} / \mathrm{dl}$ ) undergoing percutaneous coronary intervention (Group II). ACS patients not known to be diabetic undergoing percutaneous coronary intervention with normal renal function were included in the study. Patients with known Diabetes Mellitus, $\mathrm{Hb} \%<10$ $\mathrm{gm} / \mathrm{dl}$, serum creatinine $\geq 1.5 \mathrm{mg} / \mathrm{dl}$, history of intake of nephrotoxic drugs in previous 7 days, history of intravascular administration of an iodinated contrast medium in previous 7 days, severe concomitant diseases (e.g. chronic liver disease, known neoplastic disorder), hemodynamically unstable patients, patients with congestive heart failure (NYHA class III and IV) were excluded from the study.

Study procedure: Informed written consent was taken from each patient before enrollment. 
Meticulous history was taken and clinical examination was performed. Demographic data such as age, sex, height $(\mathrm{cm})$, weight $(\mathrm{kg})$, $\operatorname{BMI}\left(\mathrm{kg} / \mathrm{m}^{2}\right)$ were noted. Risk factors profiles including hypertension, dyslipidemia, family history of coronary artery disease and smoking were noted. Base line investigations, serum lipid profile, ECG, Echocardiography, and all other investigations required before percutaneous coronary intervention were done in all cases. Procedure was performed within index hospital admission with ACS. On admission random blood glucose was measured. Non- ionic low osmolar contrast agents (lopamidol) was used in all patients. Volume of contrast medium (ml) was recorded. After percutaneous coronary intervention, every patient was followed up by clinical examination and investigation. 24 hours urine volume was measured at day 1 and day 2 .
Serum creatinine, serum electrolytes was measured and creatinine clearance rate was determined within 24 hours before PCI and day 1 and 2 after PCI. In case of renal impairment (CIN) serum creatinine, serum electrolytes, and creatinine clearance rate were measured daily from $3^{\text {rd }}$ day onward after PCI until recovery. Patients were observed and questioned regarding adverse events and were instructed to report any symptoms. All adverse events were recorded during the follow up period. All relevant data were colleted in an approved data collection form.

\section{Observation and Results:}

Demographic profile of both groups were shown in the tables I \& II which revealed there was no significant difference in term of age, sex, and risk factor profiles in both study group.

Table-I

Age distribution of the study subjects $(n=100)$

\begin{tabular}{|c|c|c|c|c|c|}
\hline \multirow[t]{2}{*}{ Age in years } & \multicolumn{2}{|c|}{ Group I $(\mathrm{n}=50)$} & \multicolumn{2}{|c|}{ Group II $(\mathrm{n}=50)$} & \multirow[t]{2}{*}{$\bar{p}$ value } \\
\hline & Number & $(\%)$ & Number & $(\%)$ & \\
\hline$\leq 40$ & 4 & $(8.0)$ & 8 & $(16.0)$ & \\
\hline $41-50$ & 12 & $(24.0)$ & 11 & $(22.0)$ & \\
\hline $51-60$ & 25 & $(50.0)$ & 18 & $(36.0)$ & \\
\hline $61-70$ & 7 & (14.0) & 9 & $(18.0)$ & \\
\hline$>70$ & & $(4.0)$ & 4 & $(8.0)$ & \\
\hline $\begin{array}{l}\text { Mean } \pm \text { SDRange } \\
(\min -\max )\end{array}$ & \multicolumn{2}{|c|}{$54.6 \pm 10.3(28-85)$} & \multicolumn{2}{|c|}{$53.5 \pm 11.5(30-80)$} & $0.59^{\mathrm{ns}}$ \\
\hline
\end{tabular}

$\mathrm{ns}=$ Not significant $\mathrm{p}$ value reached from unpaired $\mathrm{t}$-test

Table-II

Distribution of clinical subsets of patients with ACS $(n=100)$

\begin{tabular}{lcccccc}
\hline Types of ACS & \multicolumn{2}{c}{ Group I $(\mathrm{n}=50)$} & & \multicolumn{2}{c}{ Group II $(\mathrm{n}=50)$} & $\mathrm{p}$ value \\
\cline { 2 - 3 } & Number & $(\%)$ & & Number & $(\%)$ & \\
\hline UA & 6 & $(12.0)$ & & 5 & $(10.0)$ & $0.74^{\text {ns }}$ \\
NSTEMI & 12 & $(24.0)$ & & 10 & $(20.0)$ & $0.62^{\text {ns }}$ \\
STEMI & 32 & $(64.0)$ & 35 & $(70.0)$ & $0.28^{\text {ns }}$ \\
\hline
\end{tabular}

ns $=$ Not significant. Data were analyzed using Pearson Chi-Square $\left(\div^{2}\right)$ test.

Table-III

Comparison of volume of contrast agent used between two groups $(n=100)$

\begin{tabular}{|c|c|c|c|c|c|}
\hline \multirow{2}{*}{$\begin{array}{l}\text { Volume of } \\
\text { contrast }(\mathrm{ml})\end{array}$} & \multicolumn{2}{|c|}{ Group I $(n=50)$} & \multicolumn{2}{|c|}{ Group II (n =50) } & \multirow[t]{2}{*}{$\mathrm{p}$ value } \\
\hline & Number & $(\%)$ & Number & $(\%)$ & \\
\hline d" 150 & 15 & $(30.0)$ & 18 & $(36.0)$ & $0.61^{\mathrm{ns}}$ \\
\hline$>150$ & 35 & $(70.0)$ & 32 & $(64.0)$ & \\
\hline
\end{tabular}

$\mathrm{s}=$ Significant. Data were analyzed using Pearson Chi-Square $\left(\div^{2}\right)$ test. 
The table II shows the clinical types of ACS between the study groups. ACS types demonstrates that $12 \%$ of patients in group I had UA, 24\% NSTEMI, and 64\% STEMI. In group II, $10 \%$ of the patients had UA, $20 \%$ NSTEMI and $70 \%$ STEMI. No significant difference was observed between the groups in terms of ACS types $(\mathrm{p}>0.05)$.
Table III shows that in group I, 30\% of patients received d" $150 \mathrm{ml}$ of contrast volume and 70\% patients received $>150 \mathrm{ml}$ of contrast. But in group II, $36 \%$ of patients received $\leq 150 \mathrm{ml}$ of contrast volume and $64 \%$ patients received $>150$ $\mathrm{ml}$ of contrast. The volume of contrast used had no significant difference between the groups $(p>0.05)$.

\section{Table-IV}

Changes in serum creatinine between baseline and day 2 among patients of study group ( $n=100)$

\begin{tabular}{lccc}
\hline Group & \multicolumn{2}{c}{ Serum creatinine } & p value \\
\cline { 2 - 3 } & Baseline & Day 2 & \\
& Mean \pm SD & Mean \pm SD & \\
\hline Group I $(\mathrm{n}=50)$ & $1.0 \pm 0.1$ & $1.1 \pm 0.1$ & $0.18^{\mathrm{ns}}$ \\
Group II $(\mathrm{n}=50)$ & $1.0 \pm 0.2$ & $1.3 \pm 0.5$ & $0.001^{\mathrm{s}}$ \\
\hline
\end{tabular}

$\mathrm{s}=$ Significant. $\mathrm{ns}=$ Not significant. Data were analyzed using paired student $\mathrm{t}$ - test.

Table-V

Changes in creatinine clearance rate $(\mathrm{CCr})$ between baseline and day 2 among patients of study group $(n=100)$

\begin{tabular}{lccc}
\hline Group & \multicolumn{2}{c}{ CCr } & p value \\
\cline { 2 - 3 } & $\begin{array}{c}\text { Baseline } \\
\text { Mean } \pm \text { SD }\end{array}$ & $\begin{array}{c}\text { Day } 2 \\
\text { Mean } \pm \text { SD }\end{array}$ & \\
\hline Group I $(\mathrm{n}=50)$ & $77.6 \pm 18.4$ & $71.6 \pm 15.9$ & $0.11^{\mathrm{ns}}$ \\
Group II $(\mathrm{n}=50)$ & $80.2 \pm 19.8$ & $67.3 \pm 21.9$ & $0.001^{\mathrm{s}}$ \\
\hline
\end{tabular}

$\mathrm{s}=$ Significant. Data were analyzed using paired student $\mathrm{t}$ - test.

Table-VI

Incidence of contrast induced nephropathy among studied patients $(n=100)$

\begin{tabular}{lcccccc}
\hline Group & \multicolumn{3}{c}{ CIN } & & p value \\
\cline { 2 - 3 } & \multicolumn{2}{c}{ Developed } & & \multicolumn{2}{c}{ Not developed } & \\
\cline { 2 - 3 } \cline { 5 - 7 } & Number & $(\%)$ & & Number & $(\%)$ & \\
\hline Group I $(\mathrm{n}=50)$ & 2 & $(4.0)$ & & 48 & $(96.0)$ & $0.004^{\mathrm{s}}$ \\
Group II $(\mathrm{n}=50)$ & 12 & $(24.0)$ & & 38 & $(76.0)$ & \\
\hline
\end{tabular}

Data were analyzed using Pearson Chi-Square $\left(\div^{2}\right)$ test.

Table-VII

Changes in serum creatinine between baseline and day 2 among patients of CIN ( $n=14)$

\begin{tabular}{lccc}
\hline Group & \multicolumn{2}{c}{ Serum creatinine } & p value \\
\cline { 2 - 3 } & Baseline & Day 2 & \\
\hline Group I $(\mathrm{n}=2)$ & $0.75 \pm 0.21$ & Mean $\pm \mathrm{SD}$ & $0.04^{\mathrm{s}}$ \\
Group II $(\mathrm{n}=12)$ & $0.96 \pm 0.25$ & $1.4 \pm 0.00$ & $0.001^{\mathrm{s}}$ \\
\hline
\end{tabular}

$\mathrm{ns}=$ Not significant. $\mathrm{s}=$ Significant. Data were analyzed using paired students $\mathrm{t}$ - test. 
The table IV shows the changes in the serum creatinine between baseline and day 2 among patients of study groups. The mean serum creatinine of group I was $1.0 \mathrm{mg} / \mathrm{dl}$ and $1.1 \mathrm{mg} / \mathrm{dl}$ at baseline and day 2 respectively, which was not statistically significant $(\mathrm{p}=0.18)$. But in group II the mean serum creatinine was $1.0 \mathrm{mg} / \mathrm{dl}$ and 1.3 $\mathrm{mg} / \mathrm{dl}$ on baseline and day 2 respectively. This differences were statistically significant $(\mathrm{p}=0.001)$.

The table $\mathrm{V}$ shows the changes in the creatinine clearance rate among patients of study groups at different time interval. The mean $\mathrm{CCr}$ of group I was $77.6 \mathrm{ml} / \mathrm{min}$ and $71.6 \mathrm{ml} / \mathrm{min}$ at baseline and day 2 respectively and the change was statistically insignificant $(p=0.11)$. It was also observed that the mean $\mathrm{CCr}$ in group II was $80.2 \mathrm{ml} / \mathrm{min}$ and $67.3 \mathrm{ml} / \mathrm{min}$ at baseline and day 2 respectively. This difference was statistically significant $(p=0.001)$.

The table VI shows when the most common definition of contrast induce nephropathy (as an increase in serum creatinine concentration e"0.5 $\mathrm{mg} / \mathrm{dl}$ or e" $25 \%$ increase of serum creatinine from baseline within 48 hours after exposure to contrast media) was used the it was observed that CIN was higher in group II (24\%) than that of group I $(4 \%)$ which was statistically significant $(\mathrm{p}=0.004)$.

The table VII shows the peak increase in the serum creatinine concentration among patients of CIN within 2 days of contrast administration, which was $0.65 \mathrm{mg} / \mathrm{dl}$ in group I as compared with $0.94 \mathrm{mg} / \mathrm{dl}$ in group II respectively. A statistically significant change in serum creatinine was observed on day 2 from baseline both in normal and high blood glucose group flowing PCI $(p<0.05)$

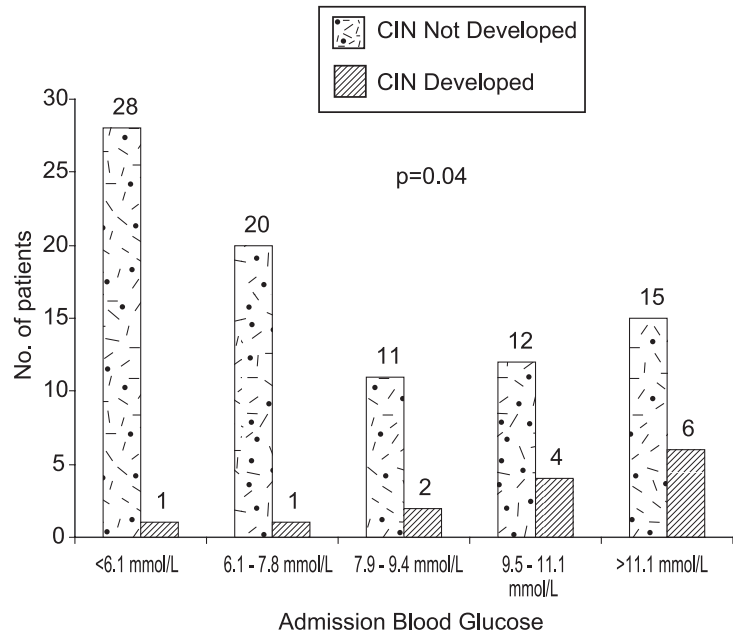

Fig.-1: Comparison between level of admission blood glucose and incidence of CIN among patients $(n=100)$.
The figure 1 shows comparison between level of admission blood glucose and incidence of CIN among ACS patients undergoing PCI not known to be diabetic. It was observed that gradual incremental increase in risk of CIN associated with higher admission blood glucose levels.

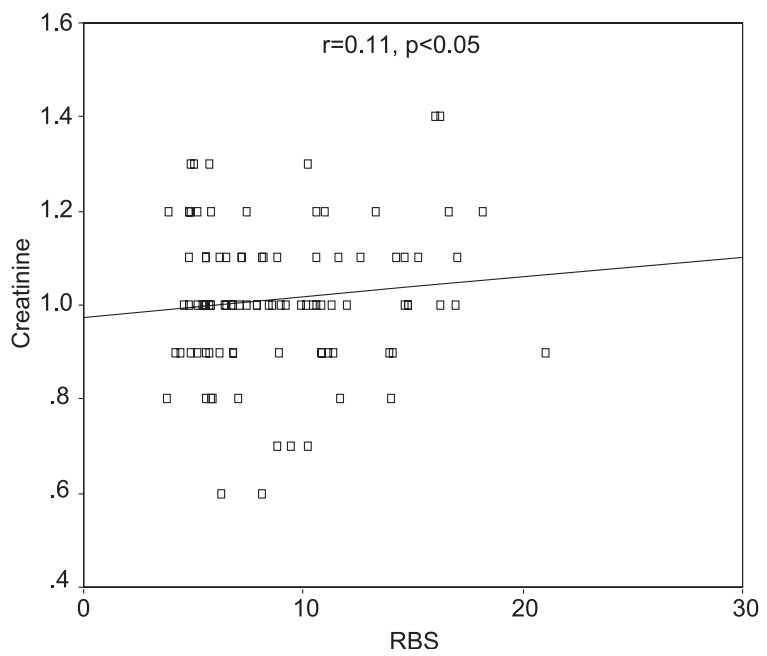

Fig.-2: Correlation between S. creatinine and admission blood glucose after PCI in ACS patients not known to be diabetic.

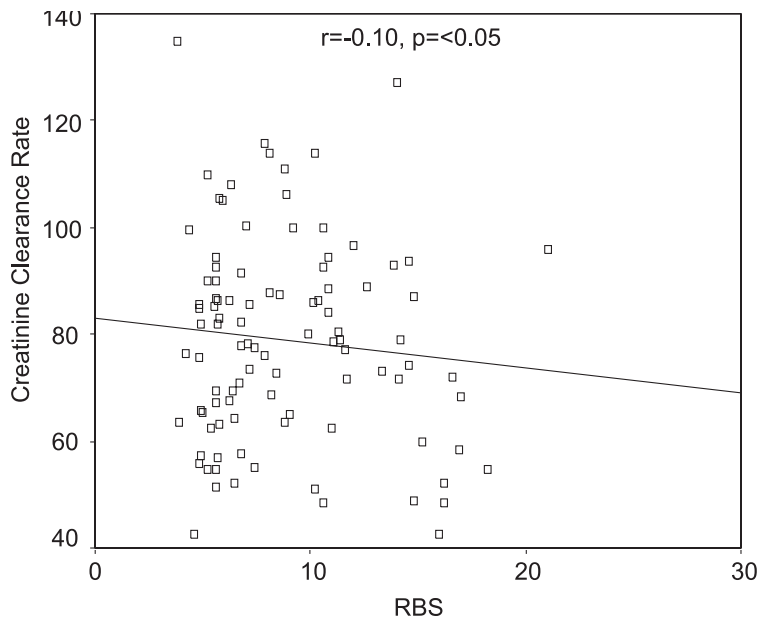

Fig.-3: Correlation between $\mathrm{CCr}$ and admission blood glucose after PCI in ACS patients not known to be diabetic.

Multiple regression analysis showed that of the 5 variables contrast induced nephropathy were found to be significantly associated with blood glucose (standardized coefficient (â) 1.580, 95\% confidence interval 1.765-13.349, $\mathrm{p}<0.05$ ) and contrast volume (standardized coefficient (â) $0.357,95 \%$ confidence interval 0.999-1.047, $\mathrm{p}<0.05)$. 


\section{Discussion:}

This was a prospective observational study conducted in the National institute of Cardiovascular Diseases (NICVD), Dhaka for a period of July, 2011 to may, 2012. A total of one hundred patients, divided into two groups, were studied during the study period over one year. Group 1(n=50) Patients with normal blood glucose $(\leq 7.8 \mathrm{mmol} / \mathrm{l}$ or $\leq 140 \mathrm{mg} / \mathrm{dl})$ and group II $(n=50)$ patients with high blood glucose $(>7.8$ $\mathrm{mmol} / \mathrm{l}$ or $>140 \mathrm{mg} / \mathrm{dl})$. The mean age of patients in group- 1 was $54.6 \pm 10.3$ years, where as in group-II it was $53.5 \pm 11.5$ years. The mean difference was not statistically significant $(p=0.59)$. Among all study patients, highest number of patients was in the age group 51-60. That is similar to study conducted by Hossain. ${ }^{10}$ The mean age of the study patients was also within the range (40-60 years) found in one study conducted in Bangladesh. ${ }^{18}$ Hayder and Shaheen observed the mean age was a little lesser than the present study. ${ }^{9,19}$ However and have observed higher mean age which may be due to increased life expectancy in western country. ${ }^{20,21}$ The majority of patient were male $82 \%$ in group I and $88 \%$ in group II and remaining female, with a male to female ratio $5.66: 1$ in the whole study population No significant $(p>0.05)$ difference was found regarding sex distribution between two groups. Almost similar male female ratio $(6.9: 1)$ was observed in a study conducted by M Akhtaruzzaman in NICVD. ${ }^{15}$ The number of female patients were less in almost all previous studies like Hossain, and Hayder Solomon, et al and McCullough, et al also observed male patients predominant in their studies. ${ }^{12,14,19,22}$

Among all ACS patients, Unstable angina was $12 \%$, NSTEMI was $24 \%$ and STEMI was $64 \%$ in group I patients. Whereas in group II 10\% patients had unstable angina $20 \%$ had NSTEMI and $70 \%$ patients had STEMI. No significant difference was observed regarding clinical diagnosis between the groups $(p=0.74$, $\mathrm{p}=0.062, \mathrm{p}=0.28$ respectively). Hossain also found less number of unstable angina patients than myocardial infarction in his study. ${ }^{12} \mathrm{But}$ Nikolsky, et al. found about half of the patients had unstable angina. ${ }^{23}$ The volume of contrast administered (£ 150ml), in normal blood glucose group was $30 \%$ and high blood glucose group was
$36 \%$ but $>150 \mathrm{ml}$ of contrast was administered $70 \%$ in normal blood glucose group and $64 \%$ in high blood glucose group. The difference in receiving contrast volume between two groups was not statistically significant $(\mathrm{P}>0.05)$. The results are consistent with Shaheen. ${ }^{9}$ But higher amount of contrast were used by Marenzi, et al and Nikolsky. ${ }^{24,25}$

When the most common definition of contrast induced nephropathy (as an increase in the serum creatinine concentration e" $0.5 \mathrm{mg} / \mathrm{dl}$ or e" $25 \%$ from baseline value at 48 hours after exposure to contrast media) was used the incidence of CIN was $24 \%$ in high blood glucose group and $4 \%$ in the normal blood glucose group that includes 12 patients in high blood glucose group and 2 patients in normal blood glucose group. The result was statistically significant $(p=0.004)$. Stolker, et al also found development of CIN is more common among hyperglycemic non diabetic ACS patients in comparison to normoglycemic non diabetic ACS patients. (18,1\% vs 51.6\%). ${ }^{17}$ Shaheen and M Akhtaruzzaman also found increased incidence of CIN among patients with high blood glucose but they included both diabetic and non diabetic patients in study population. ${ }^{9,15}$ When all study patients in both groups were considered, 14 patients developed CIN i.e the overall incidence of CIN was found $14 \%$ in the present study. The finding of the present study were very close to those of other multiple studies on contrast nephropathy. Marenzi, et al. found overall $14.5 \%$ patient developed contrast induced nephropathy, among them CIN occurred in $27 \%$ patients with acute hyperglycemia. ${ }^{24}$ Nikolsky, et al. found the incidence of CIN after percutaneous coronary intervention was $13.9 \% .{ }^{23}$ Shaheen found the incidence of CIN after coronary angiography and percutaneous coronary intervention was $10 \% .{ }^{9} \mathrm{McCullough}$, et al. also stated that incidence of CIN can rise to $50 \%$ or more in patients with multiple risk markers. ${ }^{4}$

A significant change in serum creatinine was observed on day 2 from baseline both in normal and high blood glucose group following PCI $(p<0.04$ vs. $<0.001)$. The peak increase in the serum creatinine concentration among patients of CIN within two days after administration of contrast 
medium was $0.65 \mathrm{mg} / \mathrm{dl}$ in group-I as compared with $0.94 \mathrm{mg} / \mathrm{dl}$ in group-II.Our study found that, among patients with contrast induced nephropathy one patient had admission blood glucose $<6.1 \mathrm{mmol} / \mathrm{l}$, one patient had blood glucose within 6.1 to $7.8 \mathrm{mmol} / \mathrm{l}$, two patients were within range of 7.9 to $9.4 \mathrm{mmol} / \mathrm{l}, 4$ patients had blood glucose within 9.5 to $11.1 \mathrm{mmol} / \mathrm{l}$ and highest number of patients that is 4 patients had blood glucose above $11.1 \mathrm{mmol} / \mathrm{l}$. So, it was observed that gradual incremental increase in risk of CIN associated with higher admission blood glucose levels. In a study by Stolker, et al. reported that there was a strong association between level of blood glucose and incremental increase in contrast induced acute kidney injury risk in patients without diabetes (contrast induced acute kidney injury rates across their 5 glucose groups from lowest to highest were $8.2 \%$, $9.9 \%, 12.4 \%, 14.9 \%, 24.3 \% ; p<0.001)$ which are compatible with the present study. ${ }^{17}$

A total of 2 variables revealed to be significantly associated with the development of CIN i.e blood glucose and contrast volume $(\mathrm{p}<0.05)$.In multiple regression analyses of the 5 variables, blood glucose and contrast volume were found to be the independent predictors of CIN with ORs being 1.765 and 1.0 respectively. There was positive correlation between s. creatinine and admission blood glucose, but it showed negative correlation between creatinine clearance rate (CCr) and admission blood glucose after PCI in ACS patients not known to be diabetic.

Regarding outcome of the study patients, no patient died in the present study and no patient developed acute renal failure requiring dialysis. McCullough, et al. found the occurrence of acute renal failure requiring dialysis after coronary intervention is rare $(<1 \%)$. ${ }^{4}$ The serum creatinine of $100 \%$ patients in both groups returned to base line within two weeks. In group-II 98\% returned to base line within first week and $2 \%$ returned to base line on second week. But 100\% patients of group-I serum creatinine returned to baseline within first week. The outcomes are consistent with most studies on CIN when it was reported that CIN usually recovered within two weeks. ${ }^{25}$

\section{Conclusion:}

The present study reveals that index admission high blood glucose in acute coronary syndrome patients not known to be diabetic is associated with increased incidence of contrast induced nephropathy after percutaneous coronary intervention. So, more attention should be paid to the blood glucose level of the patient before carrying out any coronary intervention.

\section{Limitations of the Study:}

Despite exercise of utmost caution through out the study, it has got some important limitation.

1) This was a prospective, observational study, not a randomized one. 2) The sample size was small, and it was a single centre based study. 3) Glycated hemoglobin estimation was not done as it is expensive, not so available and assays are not standard across laboratories. 4) Upto 2 weeks follow up was required to assess the renal status of the patients with CIN after PCI which was not done as most of the patients discharged on second or third post procedural day. 5) Creatinine clearance rate should be measured from 24 hours urinary output but here it is measured from Cockroft Gault equation to reduce cost.

\section{References:}

1. Pucelikova T, Dangas G \& Mehran R. Contrast induced nephropathy. Catheter Cardio Interv 2008; 71: 62-72.

2. Tepel M, Aspelin P and Lameire N. Contrast-induced nephropathy : A clinical and evidence-based approach. Circulation 2006; 113:1799-1806.

3. Alpert M.A and Carlino C. Pre-procedural blood glucose levels: A new risk marker for contrast-induced acute kidney injury in patients without diabetes with acute myocardial infarction. J Am Coll Cardiol 2010; 55: 14411443.

4. McCullough P.A, Adam A, Becker C.R, Davidson C, Lameire N, Stacul F\& Tumlin J. Epidemiology and prognostic implications of contrast induced nephropathy. Am J Cardiology 2006; 98: 5k-13k.

5. Capes S.E, Hunt D, Malmberg K \& Gerstein H.C. Stress hyperglycemia and increased risk of death after myocardial infarction in patients with and without diabetes: a systemic overview. The lancet 2000; 355: 773-778.

6. Petursson P, Herlitz J, Karisson T, Perers E \& Hartfold M. Admission glycaemia and outcome after acute coronary syndrome. Int J Cardiol 2007 ;116(3) :315-320.

7. Norhammer A.M, Ryden L \& Malmberg K. Independent risk factor for long term prognosis after myocardial infarction even in non diabetic patients. Diabetes Care 1999; 22:1827-1831. 
8. Mehran R and Nikolsky. Contrast-induced nephropathy: Definition, epidemiology, and patients at risk. Kidney Int $2006 ; 69$ :511-515.

9. Shaheen, Md. Nephrotoxic effects of contrast medium in patients undergoing coronary angiography and percutaneous coronary intervention. MD thesis, University of Dhaka, Bangladesh 2008.

10. Lautin E.M, Freeman N.J, Schoenfeld A.H, Bakal C.W, Haramiti N, Friedman A.C, Lautin J.L, Braha S, Kadish E.G, Sprayreyen S \& Belizon I. Radio-contrast associated renal dysfuction: A comparison of lower osmolality and conventional high-osmolality contrast media. Am J Roentgenol 1991; 157: 59-65.

11. McCullough P.A, Bertrand M.E, Brinker J.A \& Stacul F. A meta-analysis of the renal safety of iso-osmolar iodixanol compared with low-osmolar contrast media. J Am Coll Cardiol 2006 ;48: 692-699.

12. Hossain S. Comparison of nephrotoxic effect of iso-osmolar and low-osmolar non-ionic contrast media in patient with chronic kidney disease undergoing coronary angiography. Thesis (MD Cardiology), University of Dhaka, Bangladesh 2010.

13. Owen R.J, Hiremath S, Myers A, Fraser-Hill, M and Barrett B. Consensus guidelines for the prevention of contrast induced nephropathy. Can Assoc Radiol J 2011.

14. McCullough P.A, Adam A, Becker C.R, Davidson C, Lameire N, Stacul F, Tumlin J. Risk prediction of contrast induced nephropathy. Am J Cardiol 2006; 98: 27k-36k.

15. Akhtaruzzaman Md. Impact of low hemoglobin on contrast-induced nephropathy after percutaneous coronary intervention. Thesis (MD Cardiology), University of Dhaka, Bangladesh 2010.

16. Deedwania P, Kosoborod M, Barrett E, Ceriello A, Isley W, Mazzone T and Raskin P. Hyperglycemia and Acute Coronary Syndrome: A Scientific Statement from the American Heart Association Diabetes Committee of the Council on Nutrition, Physical Activity, and Metabolism. Circulation 2008; 117: 1610-16119.

17. Stolker J.M, McCullough P.A, Rao S, Inzucchi S.E, Spertus J.A, Maddox T.M, Masoudi F.A, Xiao L, Mikhail
K. Pre-procedural glucose levels and the risk for contrastinduced acute kidney injury in patients undergoing coronary angiography. J Am Coll Cardiol 2010; 55(14) :1433-1440.

18. Ali M, Reza S.A, Uddin M.J, Rahman S, Rahman A, Momenuzzama N.A.M, Ahsan S.A, Alam M.R, Hossain S.M \& Ahmed A.F.K, In-hospital outcome of 500 cases of Percutaneous Coronary Intervention. Bangladesh Heart Journal 2006; 21(1): 29-34.

19. Hayder, S. Comparison of ionic and nonionic contrast media in cardiac angiography; clinical, electrocardiographic and haemodynamic changes. Thesis (MD Cardiology), University of Dhaka, Bangladesh 1999.

20. Rudnick M.R, Goldfarb S, Wexler L, Ludbrook P.A, Murphy P.J, Halpern E.F, Hill, J.A, Winniford M, Cohen M.B, VanFossen D.B. Nephrotoxicity of ionic and nonionic contrast media in 1196 patients: a randomized trial. The iohexol cooperative study. Kidney Int 1995; 47: 254-261.

21. Kini A.S, Mitre C.A, Kamran M, Suleman J, Kim M, Duffy M.E, Marmur J.D \& Sharma S.K. Changing trends in incidence and predictors of radiographic contrast nephropathy after percutaneous coronary intervention with use of fenoldopam. Am J Cardiol 2002; 89: 999-1002.

22. Solomon R. The role of osmolality in the incidence of contrast-induced nephropathy : A systemic review of angiographic contrast media in high risk patients. Kidney Int 2005; 68: 2256-2263.

23. Nikolsky E, Mehran R, Lasic Z, Mintz G.Z, Lansky A.J \& Dangas G. Low-haematocrit predicts contrast-induced nephropathy after percutaneous coronary intervention. Kidney Int 2005; 67:706-713.

24. Marenzi G, Assanelli E, Marana I, Lauri G, Campodonico J, Grazi M, Metrio M.D, Galli S, Fabbiocchi F, Montorsi P, Vaglia F \& Bartorelli A.L. N-acetylcystine and contrastinduced nephropathy in primary angioplasty. $N$ Eng $J \mathrm{Med}$ 2006; .354:2773-2782.

25. Goldenberg I \& Matetzky S. Nephropathy induced by contrast media: Pathogensis, risk factors and preventive strategies. Can Med Assoc J 2005; 172(11): 1461-1471. 